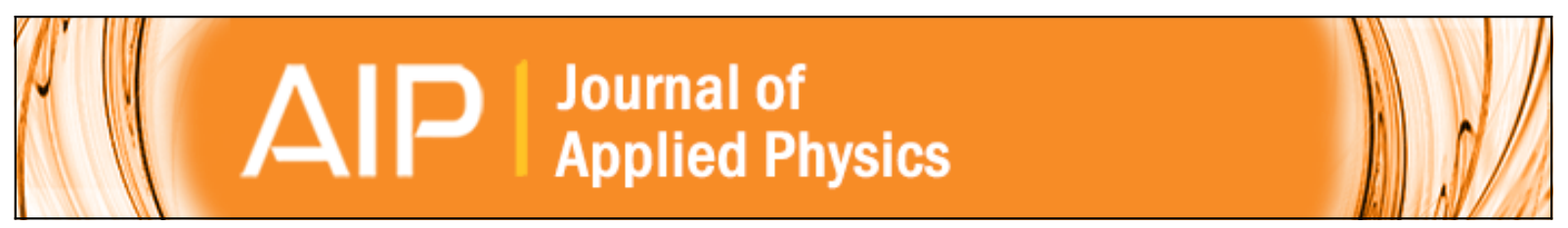

\title{
Metallic nanoparticles grown in the core of femtosecond laser micromachined waveguides
}

J. M. P. Almeida, P. H. D. Ferreira, D. Manzani, M. Napoli, S. J. L. Ribeiro, and C. R. Mendonça

Citation: Journal of Applied Physics 115, 193507 (2014); doi: 10.1063/1.4875485

View online: http://dx.doi.org/10.1063/1.4875485

View Table of Contents: http://scitation.aip.org/content/aip/journal/jap/115/19?ver=pdfcov

Published by the AIP Publishing

\section{Articles you may be interested in}

Er:Yb-doped oxyfluoride silicate glass waveguide amplifier fabricated using femtosecond laser inscription

Appl. Phys. Lett. 90, 131102 (2007); 10.1063/1.2716866

Fabrication of waveguides in Foturan by femtosecond laser

J. Laser Appl. 18, 320 (2006); 10.2351/1.2355525

Densification of silica glass induced by 0.8 and $1.5 \mu \mathrm{m}$ intense femtosecond laser pulses

J. Appl. Phys. 99, 093104 (2006); 10.1063/1.2196237

Optical waveguide fabrication with double pulse femtosecond lasers

Appl. Phys. Lett. 86, 251103 (2005); 10.1063/1.1954879

Waveguide structures in heavy metal oxide glass written with femtosecond laser pulses above the critical selffocusing threshold

Appl. Phys. Lett. 86, 121109 (2005); 10.1063/1.1888032

\section{AIP $\mid$ chaos CALL FOR APPLICANTS Seeking new Editor-in-Chief}




\title{
Metallic nanoparticles grown in the core of femtosecond laser micromachined waveguides
}

\author{
J. M. P. Almeida, ${ }^{1}$ P. H. D. Ferreira, ${ }^{1}$ D. Manzani, ${ }^{2}$ M. Napoli, ${ }^{2}$ S. J. L. Ribeiro, ${ }^{2}$ \\ and C. R. Mendonça ${ }^{1, a)}$ \\ ${ }^{1}$ Instituto de Física de São Carlos, Universidade de São Paulo-USP, CP 369, São Carlos, \\ Sao Paulo 13560-970 Brazil \\ ${ }^{2}$ Institute of Chemistry-São Paulo State University-UNESP, CP 355, Araraquara, Sao Paulo 14801-970, \\ Brazil
}

(Received 29 March 2014; accepted 26 April 2014; published online 20 May 2014)

\begin{abstract}
3D-waveguides containing silver nanoparticles have been fabricated in tungsten leadpyrophosphate glass by femtosecond laser micromachining. Nucleation and growth of nanoparticles occur in a single step process when high repetition rate laser $(\mathrm{MHz})$ is employed, while an additional annealing is required for the irradiation using $\mathrm{kHz}$ laser system. The presence of nanoparticles locally changes the refractive index, and, therefore, the elliptical structures produced by direct laser writing were able to guide light. By increasing the pulse energy applied during the micromachining, the waveguide size increased from 2 to $30 \mu \mathrm{m}$, while their propagation loss decrease from 1.4 to $0.5 \mathrm{~dB} / \mathrm{mm}$ at $632.8 \mathrm{~nm}$. (C) 2014 AIP Publishing LLC.
\end{abstract}

[http://dx.doi.org/10.1063/1.4875485]

\section{INTRODUCTION}

Glasses are promising candidates for the development of technological applications on the account of their relatively easy manipulation, which makes it possible to fabricate them in different sizes, shapes, and compositions. Among these features, the ability of changing the glass composition can be pointed out as one of the most relevant, once it determines the material properties and performance. ${ }^{1}$ Thus, several glass-formers and modifiers have been exploited for new technologies in optics and photonics. For instance, tungstatephosphate glasses have been demonstrated to be a promising material due to their attractive linear and nonlinear optical properties. Their $\mathrm{P}-\mathrm{O}-\mathrm{W}$ bond results in a highly connected network, which is also favorable for further addition of $\mathrm{WO}_{3}$ that increases the polarizability, and consequently, the optical nonlinearities of the glass matrix. ${ }^{2}$ In the same direction, the addition of heavy metal oxide also increases the material's nonlinearity, a desirable feature for photonic devices. Lead oxide has been a great glass modifier, providing suitable figures of merit for all-optical switching, $\mathrm{n}_{2} / \beta \lambda>1$, in which $\mathrm{n}_{2}$ is the nonlinear index of refraction and $\beta$ the two-photon absorption coefficient. ${ }^{3-5}$ Tungsten leadpyrophosphate glasses have demonstrated an ultra-fast electronic (100-200 fs) third-order nonlinearity with a nonlinear index of refraction one order of magnitude higher than fused silica. ${ }^{4,6}$ Furthermore, this glass matrix can be successfully used to form metallic nanoparticles, upon adding the desired metal to the matrix, affecting its nonlinearities at the plasmon band region. ${ }^{6}$

Despite the good features displayed by tungsten leadpyrophosphate glasses, they have not yet been exploited for the development of device applications. To accomplish this

a)crmendon@ifsc.usp.br task, the choice of the proper material processing method is crucial. Among the techniques for the fabrication of photonic devices, femtosecond laser micromachining has unique advantages that enable processing the material's bulk within the micrometer scale, without damaging its surface. ${ }^{7}$ In addition, as a consequence of the nonlinear interaction during micromachining, the optical properties can be locally changed, resulting in different absorption coefficient, refractive index, and structure. ${ }^{8}$ For instance, the spatially controlled growth of metallic nanoparticles in glass using femtosecond laser irradiation has been demonstrated. ${ }^{9-12}$ The control of such properties in a confined region makes possible the development of photonic devices, as waveguides, splitters, resonators, and microfluidic channels. ${ }^{7,8}$

Fabrication of waveguides in materials with promising optical nonlinearities has been the subject of several studies aiming at integrated photonic devices. Most of these studies, however, concern planar structures, in which three or more layers of thin films are deposited on substrates by conventional methods, such as chemical vapor deposition, ionexchange, and sol-gel. ${ }^{13}$ On the other hand, by using fs-laser micromachining, direct writing of 3D-waveguides in glass can be realized. Glass composition, laser parameters and experimental settings, i.e., wavelength, repetition rate, numerical aperture, and scan speed, affect the waveguide features. Hence, depending on the experimental conditions, fs-laser micromachining can lead to waveguides with refractive index changes from $10^{-4}$ to $10^{-2}$, resulting in propagation of fundamental or higher-order modes, and loss ranging from 0.7 to $4.0 \mathrm{~dB} / \mathrm{cm} .^{7,8,14-16}$ In this direction, the purpose of this paper is to encompass the interesting nonlinear optical properties of tungsten lead-pyrophosphate glass and femtosecond laser micromachining to produce waveguides containing metallic nanoparticles in its core, using one-step laser processing material. 


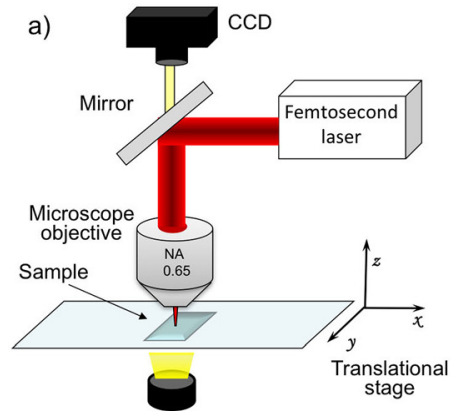

b)

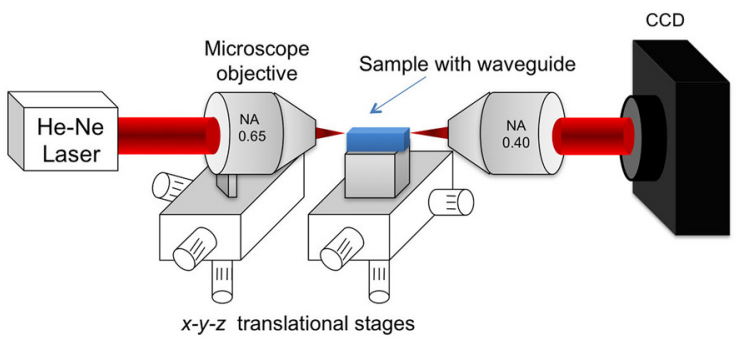

FIG. 1. Experimental setup used for (a) femtosecond laser micromachining used to produce waveguide and generate Ag NPs; and (b) waveguide characterization at $632.8 \mathrm{~nm}$.

\section{EXPERIMENTAL}

The preparation method, structure, thermal and optical properties of the $70 \mathrm{~Pb}_{2} \mathrm{P}_{2} \mathrm{O}_{7}-30 \mathrm{WO}_{3}(\mathrm{~mol} . \%)$ glass were recently described in Ref. 2. Its refractive index at $633 \mathrm{~nm}$, glass transition and crystallization temperatures are 1.9, $432^{\circ} \mathrm{C}$, and $520^{\circ} \mathrm{C}$, respectively. This sample was doped with $1 \mathrm{~mol} . \%$ of $\mathrm{AgCl}$ during the melting, in order to obtain metallic silver nanoparticles (Ag NPs) by fs-laser micromachining.

Two distinct Ti:Sapphire lasers were used in this study: (i) An amplified system with repetition rate of $1 \mathrm{kHz}$, pulse energy ( $\mathrm{E}_{\mathrm{p}}$ ) of $400 \mu \mathrm{J}$ and $150 \mathrm{fs}$; and (ii) an oscillator operating at $5 \mathrm{MHz}$ with pulses of $100 \mathrm{~nJ}$ and $50 \mathrm{fs}$, both centered at $800 \mathrm{~nm}$. The laser pulses were focused beneath the sample surface by a microscope objective $(\mathrm{NA}=0.65)$, while the sample was moved at constant speed in the plane perpendicular to the laser propagation (Fig. 1(a)). Thus, waveguides were written throughout the sample. The formation of Ag NPs was verified using linear absorption spectroscopy (Shimadzu UV-1800) and transmission electron microscopy-TEM (Tecnai G2 F20-200 kV) performed in a grating pattern (160 lines, $3 \mathrm{~mm}$ long, separated by $15 \mu \mathrm{m}$ ) produced by direct laser writing using pulse energy of $30 \mathrm{~nJ}$ and an scan speed of $100 \mu \mathrm{m} / \mathrm{s}$.

A standard optical coupling system, based on a He-Ne laser $(632.8 \mathrm{~nm})$ and microscope objectives (Fig. 1(b)), was used to couple and observe the light guiding. Waveguide losses were determined by measuring the input and output power at the entrance of the first objective and right after an iris placed at the exit of the second objective, taking into account the transmission of all components of the system. In order to distinguish coupling from propagation losses, the transmittance was obtained for a waveguide of $5.7 \mathrm{~mm}$ and then for half of such length. Near-field propagation mode was collected onto a CCD and analyzed in an image software.

\section{RESULTS AND DISCUSSIONS}

The absorption spectrum of the as prepared silver doped glass, displayed in Fig. 2 (curve A), shows that the sample is transparent at the visible and near infrared, where fs-laser excitation is performed. The UV absorption edge corresponds to a band gap energy of $3.2 \mathrm{eV}$. A color change was observed after the fs-laser irradiation using the oscillator system (5 MHz repetition rate), as shown in the inset of Fig. 2. Such color corresponds to a broad absorption band, centered at $470 \mathrm{~nm}$ (spectrum B), that corresponds to the surface plasmon resonance of metallic nanoparticles. It is known that the frequency, bandwidth, and intensity of the plasmon band are affected by the size, shape, and dielectric environment surrounding the nanoparticles. ${ }^{17}$ In general, the surface plasmon resonance of silver nanoparticles in oxide glass is located at $450 \mathrm{~nm} .{ }^{11}$ Thus, the absorption band at $470 \mathrm{~nm}$ in spectrum B (Fig. 2) indicates that $\mathrm{Ag}^{0}$ NPs have been generated during fs-laser irradiation. Nonetheless, we used TEM and electron diffraction to verify the presence and composition of the NPs, as illustrated in Fig. 3. Quasi-spherical NPs, with diameter around $10 \mathrm{~nm}$, were observed and the (111), (200), and (311) crystallographic planes of silver were indexed (crystmet database, \#35881). The silver doped tungsten lead-pyrophosphate glass was also micromachined using an amplified laser system, with repletion rate of $1 \mathrm{kHz}$ $(150 \mathrm{fs}, \mathrm{Ep}=380 \mu \mathrm{J})$. In this case, the irradiation resulted in an increase of the absorbance for the visible region of the spectrum $\left(\alpha=1.6 \mathrm{~cm}^{-1}\right)$ due to the formation of color center and reduction of silver ions. ${ }^{10,11,18} \mathrm{~A}$ small plasmon band was observed only after a heat treatment, preformed at $400{ }^{\circ} \mathrm{C}$ for $1 \mathrm{~h}$. The effect of color centers caused by the irradiation using the $\mathrm{MHz}$ laser can be ruled out, since the absorbance due to the plasmon band overcomes any absorption caused by the color center.

It is known that high power laser and ionizing radiation result in photoelectrons in oxide glasses, which move through the solid at high speed leading to secondary

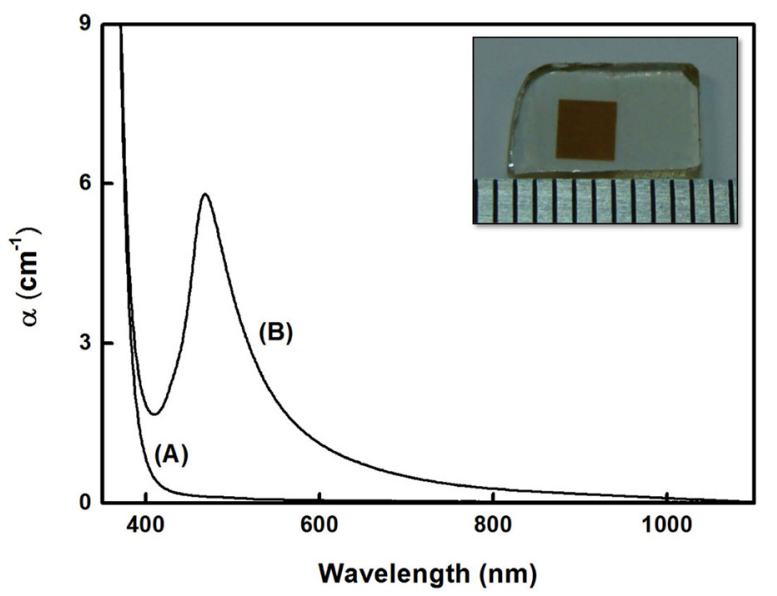

FIG. 2. Linear absorption spectrum of (A) as prepared $70 \mathrm{~Pb}_{2} \mathrm{P}_{2} \mathrm{O}_{7}-30 \mathrm{WO}_{3}$ : $1 \mathrm{AgCl}$ (mol. \%) glass; and (B) after the irradiation with fs-laser (oscillator system $-\mathrm{E}_{\mathrm{p}}=30 \mathrm{~nJ}, 50 \mathrm{fs}$, and $5 \mathrm{MHz}$ of repetition rate), using a scan speed of $100 \mu \mathrm{m} / \mathrm{s}$ to produce a grating pattern of 160 lines with $3 \mathrm{~mm}$, separated by $15 \mu \mathrm{m}$. The inset shows the difference between the irradiated and non irradiated regions of the sample. 


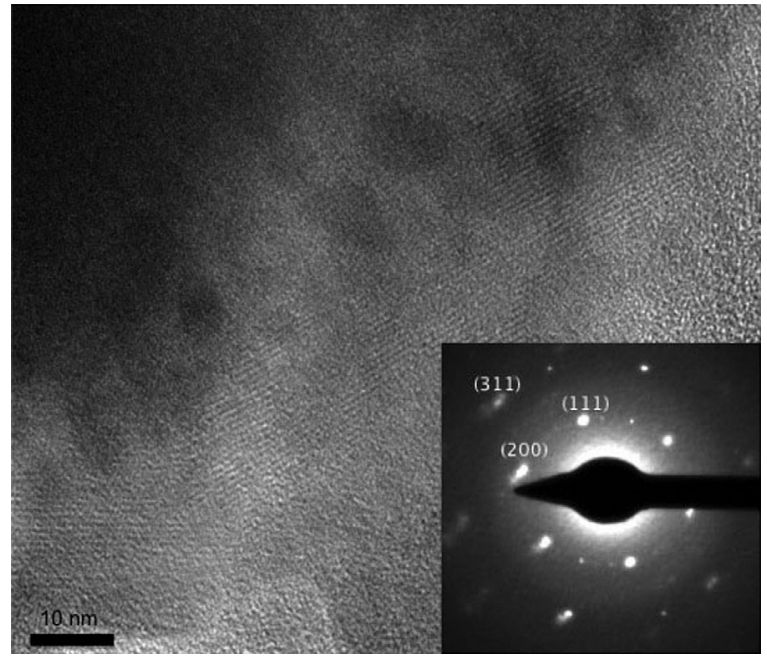

FIG. 3. TEM image and electron diffraction pattern obtained from the glass sample after irradiation with fs-laser.

electrons and holes. ${ }^{19}$ These secondary electrons and holes are trapped by defects in the glass matrix producing new electronic configurations that are understood as color centers. ${ }^{18,20}$ In the case of femtosecond lasers in transparent glasses, free electrons are generated due to nonlinear optical interactions, as photoionization and avalanche ionization. ${ }^{7,8}$ The former directly promotes electrons from the valence to the conduction band by multiphoton absorption, while avalanche ionization requires seed electrons already in the conduction band, which keep absorbing energy from the photons until it has enough energy to transfer an electron from the valence to the conduction band by collision. ${ }^{21}$ In this way, the generated electrons can be trapped (color center), or interact to the ions in the glass matrix, resulting on the photoreduction of the $\mathrm{Ag}^{+}$, which is fundamental for the nanoparticle nucleation. Finally, its growth happens due to atomic mobility promoted by some heating process. ${ }^{11}$

Although fs-lasers are known for avoiding thermal effects during the light-matter interaction, depending on the repetition rate, local heating can occur due to the cumulative effect. $^{22}$ For the oscillator system $(5 \mathrm{MHz})$, the time interval between two subsequent laser pulses is $200 \mathrm{~ns}$, that is shorter than the heat diffusion time out of the focal volume-generally $1 \mu$ s for high numerical aperture lenses. Therefore, metallic nanoparticles are generated in a one-step laser processing, since the heat promotes aggregation of the reduced metal atoms into nanoparticles. On the other hand, for the amplified system, pulses are delivered every $1 \mathrm{~ms}$, resulting on enough time to the lattice cool down before the next pulse arrives, preventing the cumulative heat effect. Thus, when lasers with $\mathrm{kHz}$ repetition rate are employed, a further annealing is required to promote the growth of nanoparticles.

The process of nanoparticle formation in glass by femtosecond laser exposure can be summarized according to the Eqs. (1)-(3), which describe the free electron generation due to nonlinear optical interactions (Eq. (1)); the photoreduction of silver ions (Eq. (2)); and the agglomeration of the neutral atoms as a consequence of heat caused by high repetition rate lasers or thermal treatment (Eq. (3)). ${ }^{23}$

$$
\begin{gathered}
\text { Glass matrix } \stackrel{h v}{\longrightarrow} \text { hole }+e^{-}, \\
A g^{+}+e^{-} \rightarrow A g^{0}, \\
n A g^{0} \stackrel{\Delta}{\longrightarrow} A g_{n} .
\end{gathered}
$$

It has been reported that the presence of silver NPs in silicate glasses leads to increase of the refractive index in $4.6 \%$ (or $\Delta \mathrm{n}=7.10^{-2}$ ). ${ }^{24}$ Thus, the micromachined structures containing Ag NPs should be able to act as waveguide. Furthermore, recent studies have suggested that high repetition rate lasers present some advantages over the low repetition rate systems, for providing more symmetric and circular structures on account of the heat deposited by successive pulses. ${ }^{14,22}$ For this reason, we have performed the light coupling experiments only in the structures fabricated with the oscillator. Images of the waveguides fabricated with $\mathrm{E}_{\mathrm{p}}=35 \mathrm{~nJ}$ and $60 \mathrm{~nJ}$, as well as their near-field distribution of the light guided at $632.8 \mathrm{~nm}$ are shown in Figs. 4 and 5, respectively. A representative top view image of the waveguides is illustrated in Fig. 4(a). We observed an increase of the waveguide size from 2 to $30 \mu \mathrm{m}$, when the pulse energy used for the micromachining was increased. The waveguide shape also depends on the pulse energy; for the lower pulse energy (Fig. 4(b)), we obtained an elliptical shape, with a dark region at the center, and a more circular structure with the dark region at the bottom for the higher energy (Fig. 5(a)). The dark region reveals a preferential aggregation of the NPs in such positions. The preferential accumulation of

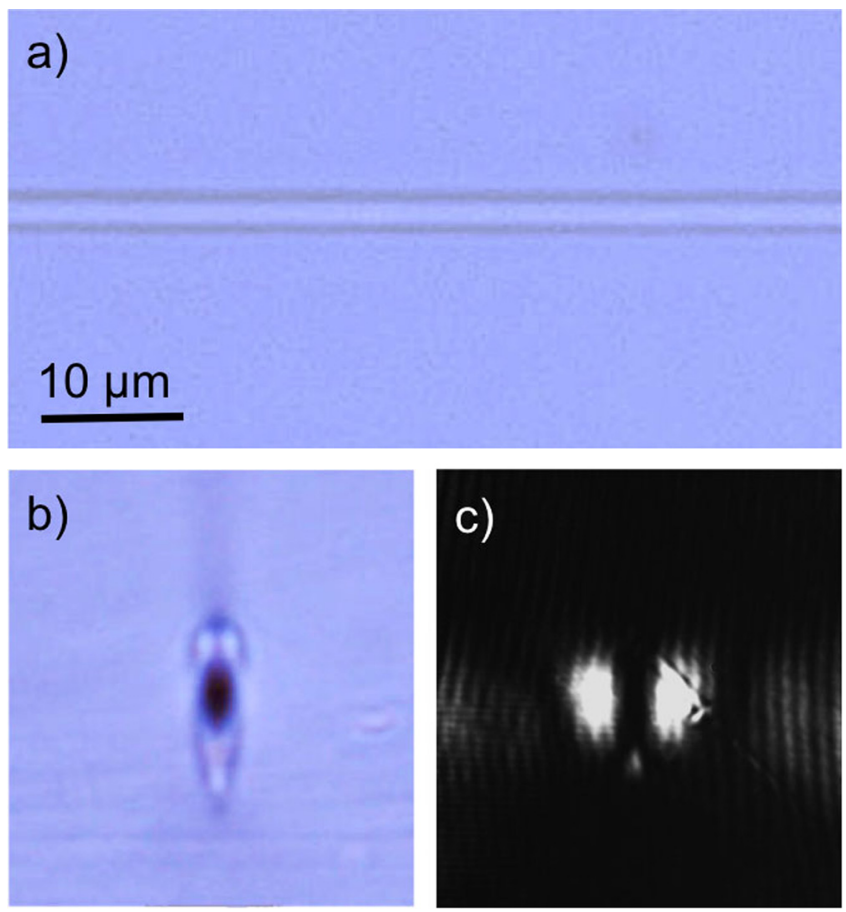

FIG. 4. Optical microscopy images of the waveguide fabricated with the oscillator laser ( $5 \mathrm{MHz}$ of repetition rate), using pulse energy of $35 \mathrm{~nJ}$, scan speed of $10 \mu \mathrm{m} / \mathrm{s}$, and NA $=0.65$. (a) and (b) show the top and end views, respectively, while (c) displays the near-field output profile of the light guided at $632.8 \mathrm{~nm}$. 

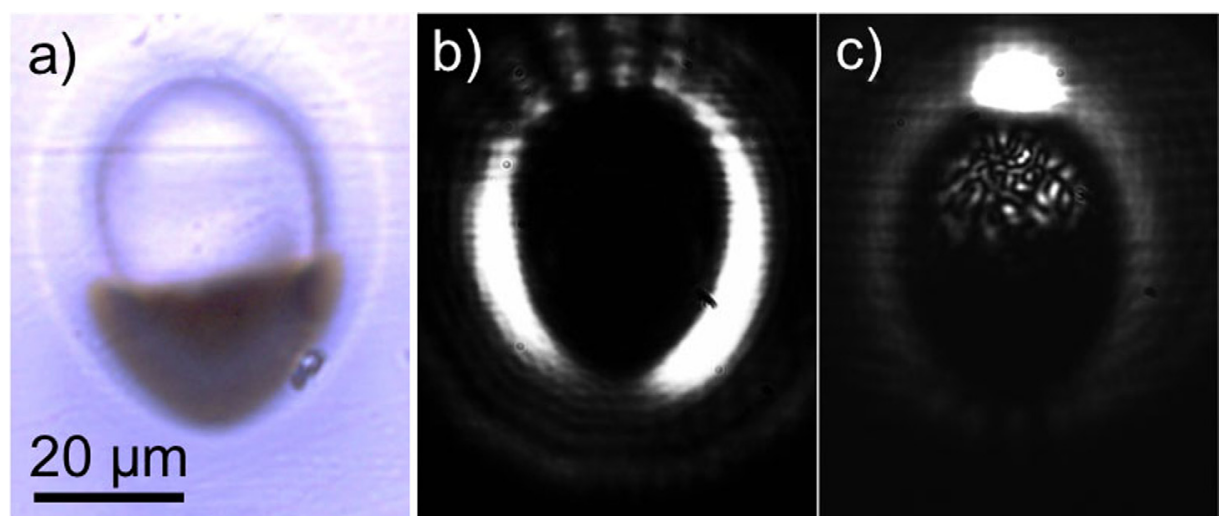

FIG. 5. (a) End view of the waveguide fabricated with pulse energy of $60 \mathrm{~nJ}$, focused $200 \mu \mathrm{m}$ bellow the sample's surface using $\mathrm{NA}=0.65$, scan speed of $10 \mu \mathrm{m} / \mathrm{s}$, and $5 \mathrm{MHz}$ of repetition rate; and (b) different light guided profiles at $632.8 \mathrm{~nm}$ obtained by changing the coupling alignment.

metallic NPs at the lower region has already been observed for silicate and borosilicate glasses shined with fs-lasers. ${ }^{10,24}$ Such behaviour might be due to the non-uniform laser intensity distribution as a consequence of the spherical aberration caused by high numerical aperture lens, ${ }^{9}$ or because of the self-focusing effect, that happens when materials with $\mathrm{n}_{2}>0$ are exposed to high laser intensities. ${ }^{10}$ However, although the dark regions present a higher amount of nanoparticles, it is reasonable to assume that in the rim outside such region, nanoparticles are also present, but in a smaller concentration, since Ag atoms could diffuse to the boundary regions due to fs-laser induced temperature gradient. ${ }^{25,26}$

Independently on the NP distribution, both structures were able to guide light at $632.8 \mathrm{~nm}$, indicating a refractive index change at the irradiated regions. As waveguides have been demonstrated in different glass due to structure modification after femtosecond laser exposure, ${ }^{8}$ it is reasonable to assume that the light confinement observed in Figs. 4(c), 5(b), and 5(c) results from a combination of the presence of nanoparticles and structural modification. Because the nanoparticle formation happens in a single step process, it is not possible to distinguish the contribution of each process on the alteration of the refractive index. Nonetheless, based on the literature, we suggest that the main contribution is caused by the nanoparticles, once $\Delta \mathrm{n}=1.4 \times 10^{-3}$ (Ref. 14) has been observed on account of structural modification whereas $7 \times 10^{-2}$ (Ref. 24) when silver nanoparticles are produced by direct laser writing in silicate glasses. In addition, no refractive index change was detected in Foturan glass due to irradiation with femtosecond laser of low energy, but a $\Delta \mathrm{n}=4 \times 10^{-3}$ was observed after further annealing as a result of silver nanoparticle formation. ${ }^{27}$

In general, the light propagation occurs at the periphery of the structures, as seen on the near-field output pattern of Figs. 4(c) and 5(b). Nonetheless, in the case of the waveguide fabricated with $\mathrm{E}_{\mathrm{p}}=60 \mathrm{~nJ}$, the light coupling also happens at the head of the structure, as shown in Fig. 5(c), indicating a non-uniform refractive index distribution. Such feature seems does not agree to the expected result, once the region with high nanoparticle contraction should present an enhancement of $\Delta \mathrm{n}$ and, therefore, higher light confinement. However, the light guided $(632.8 \mathrm{~nm})$ can be absorbed and scattered by nanoparticles. Thus, according to the increase of nanoparticles, there is a decrease of the transmittance. This trade-off between higher index of refraction and raise of absorption and scattering defines if there will be light guiding in a specific region. That is the main reason why the guiding was not observed at those regions of preferential accumulation of nanoparticles and the light confinement happens in the structure's rim, where the refractive change overcomes the absorption and scattering. We have analyzed the optical loss of those waveguides measuring their transmittance at $632.8 \mathrm{~nm}$ for the whole $(5.7 \mathrm{~mm})$ and half-length waveguide. It is difficult to report the losses with accuracy due to the high dispersion of the transmittance values, given the nature of the waveguides. Nonetheless, for the waveguide fabricated with $\mathrm{E}_{\mathrm{p}}=35 \mathrm{~nJ}$, we obtained a propagation loss of $1.4 \mathrm{~dB} / \mathrm{mm}$ and coupling loss of $9 \mathrm{~dB}$, while for the waveguide fabricated with $E_{p}=60 \mathrm{~nJ}$, we found $0.5 \mathrm{~dB} / \mathrm{mm}$ and $11 \mathrm{~dB}$, respectively. Such values correspond to an average of at least ten measurements. The differences between both waveguides are related to their sizes and refractive index distributions. Waveguides fabricated in silicate glass slides (Corning 0215) using the same method have propagation loss of about $0.3 \mathrm{~dB} / \mathrm{mm}$ at $632.8 \mathrm{~nm} .{ }^{14}$ It is important to point out that at $632.8 \mathrm{~nm}$, the glass containing silver nanoparticles has an absorption coefficient of $0.8 \mathrm{~cm}^{-1}$ (corresponding to an absorption loss $0.35 \mathrm{~dB} / \mathrm{mm}$ ) associated to the plasmon band (see Fig. 2). Thus, the optical losses can be attributed not only to the light scattering due to the roughness of the waveguide but also to absorption at $632.8 \mathrm{~nm}$. In spite of those losses, the waveguides reported herein are still interesting for photonic applications, since short optical path are required. In addition, the waveguide efficiency is expected to be better for the infrared wavelengths, where there is no absorption and scattering are minimized.

\section{CONCLUSION}

Femtosecond laser micromachining has already been used to fabricate 3D waveguide in glass as well as to grow metallic nanoparticles in controlled regions. In this paper, we have shown how both features can be encompassed to produce waveguides containing nanoparticles using one-step laser processing, and characterized the propagation mode and losses of those waveguides when low and high pulse energy is applied for the fabrication. Nucleation and growth of silver nanoparticles in lead-pyrophosphate glasses arise from the photoreduction of the Ag ions and their aggregation, as a consequence of the free electron generation and the 
local heat, provided by the irradiation with femtosecond laser pulses with repetition rate of MHz. When amplified laser system with $\mathrm{kHz}$ of repetition rate is employed, a subsequent annealing is required to grow the nanoparticles, since there is no local heat during the laser exposure to aid metal agglomeration. By increasing the pulse energy from 37 to $60 \mathrm{~nJ}$, the waveguide size increased from 2 to $30 \mu \mathrm{m}$, while their propagation loss decreased from 1.4 to $0.5 \mathrm{~dB} / \mathrm{mm}$ at $632.8 \mathrm{~nm}$. Optical microscopy images showed a preferential accumulation of nanoparticles in the center of the smaller waveguide and at the bottom of the bigger one. Such behavior is associated with the light intensity distribution profile, which can be affected by the self-focusing. Therefore, the light guiding was observed at the peripheral regions of the structures, suggesting a non-uniform refractive index distribution.

\section{ACKNOWLEDGMENTS}

The authors acknowledge the financial support from the Brazilian research funding agencies FAPESP, CAPES, and CNPq. We would also like to thank Professor C. R. M. Afonso, from Materials Engineering Department-Federal University of São Carlos, for the TEM images, and E. C. Domenicucci, from Crystal Grown and Ceramic Materials group (IFSC), for polishing the samples.

${ }^{1}$ M. Yamane and Y. Asahara, Glasses for Photonics (Cambridge University Press, Port Chester, NY, 2000).

${ }^{2}$ D. Manzani, R. G. Fernandes, Y. Messaddeq, S. J. L. Ribeiro, F. C. Cassanjes, and G. Poirier, Opt. Mater. 33, 1862 (2011).

${ }^{3}$ J. M. P. Almeida, L. De Boni, A. C. Hernandes, and C. R. Mendonca, Opt. Express 19, 17220 (2011)

${ }^{4}$ T. R. Oliveira, K. Fedus, D. Manzani, E. L. Falcao-Filho, G. Boudebs, C. B. de Araujo, and Y. Messaddeq, J. Appl. Phys. 108, 103523 (2010).

${ }^{5}$ G. T. Boyd, J. Opt. Soc. Am. B 6, 685 (1989).
${ }^{6}$ D. Manzani, J. M. P. Almeida, M. Napoli, L. D. Boni, M. Nalin, C. R. M. Afonso, S. J. L. Ribeiro, and C. R. Mendonça, Plasmonics 8, 1667 (2013).

${ }^{7}$ R. R. Gattass and E. Mazur, Nat. Photonics 2, 219 (2008).

${ }^{8}$ D. M. Krol, J. Non-Cryst. Solids 354, 416 (2008).

${ }^{9}$ J. M. P. Almeida, V. Tribuzi, R. D. Fonseca, A. J. G. Otuka, P. H. D. Ferreira, V. R. Mastelaro, P. Brajato, A. C. Hernandes, A. Dev, T. Voss, D. S. Correa, and C. R. Mendonca, Opt. Mater. 35, 2643 (2013).

${ }^{10}$ J. M. P. Almeida, L. De Boni, W. Avansi, C. Ribeiro, E. Longo, A. C. Hernandes, and C. R. Mendonca, Opt. Express 20, 15106 (2012).

${ }^{11}$ J. R. Qiu, M. Shirai, T. Nakaya, J. H. Si, X. W. Jiang, C. S. Zhu, and K. Hirao, Appl. Phys. Lett. 81, 3040 (2002).

${ }^{12}$ J. R. Qiu, X. W. Jiang, C. S. Zhu, M. Shirai, J. Si, N. Jiang, and K. Hirao, Angew. Chem.-Int. Ed. 43, 2230 (2004).

${ }^{13}$ F. Gan and L. Xu, Photonic Glass (World Scientific, Singapore, 2006).

${ }^{14}$ L. M. Tong, R. R. Gattass, I. Maxwell, J. B. Ashcom, and E. Mazur, Opt. Commun. 259, 626 (2006).

${ }^{15}$ J. Siegel, J. M. Fernandez-Navarro, A. Garcia-Navarro, V. Diez-Blanco, O. Sanz, J. Solis, F. Vega, and J. Armengol, Appl. Phys. Lett. 86, 121109 (2005).

${ }^{16}$ A. Ferrer, A. Ruiz de la Cruz, D. Puerto, W. Gawelda, J. A. Valles, M. A. Rebolledo, V. Berdejo, J. Siegel, and J. Solis, J. Opt. Soc. Am. B 27, 1688 (2010).

${ }^{17}$ U. Kreibig and M. Vollmer, Optical Properties of Metal Clusters (Springer Verlag, Berlin Heidelberg, 2010).

${ }^{18}$ A. Bishay, J. Non-Cryst. Solids 3, 54 (1970).

${ }^{19}$ R. K. Sheline, D. E. Sharp, and W. J. Arner, J. Chem. Phys. 19, 1422 (1951).

${ }^{20}$ S. G. Demos, P. R. Ehrmann, M. A. Johnson, K. I. Schaffers, A. M. Rubenchik, and M. D. Feit, Opt. Express 21, 4854 (2013).

${ }^{21}$ L. R. Cerami, E. Mazur, S. Nolte, and C. B. Schaffer, in Ultrafast Optics, edited by R. Trebino and J. Squier (Trafford Publishing, Victoria, Canada, 2007).

${ }^{22}$ C. B. Schaffer, A. Brodeur, J. F. Garcia, and E. Mazur, Opt. Lett. 26, 93 (2001).

${ }^{23}$ J. M. P. Coelho, C. Silva, A. Ruivo, and A. P. Matos, Mater. Sci. Forum 730-732, 915 (2012).

${ }^{24}$ Y. Dai, G. Yu, M. He, H. Ma, X. Yan, and G. Ma, Appl. Phys. B 103, 663 (2011).

${ }^{25}$ S. M. Eaton, H. Zhang, M. L. Ng, J. Li, W.-J. Chen, S. Ho, and P. R. Herman, Opt. Express 16, 9443 (2008).

${ }^{26}$ S. M. Eaton, H. B. Zhang, and P. R. Herman, Opt. Express 13, 4708 (2005).

${ }^{27}$ Y. Cheng, K. Sugioka, M. Masuda, K. Shihoyama, K. Toyoda, and K. Midorikawa, Opt. Express 11, 1809 (2003). 